\title{
Pseudarthrosen am Unterarm
}

Valentin Rausch, Michael Hackl, Christian Ries, Lars P. Müller

\section{Einleitung}

Unterarmfrakturen treten mit einer Häufigkeit von ca. 12,5/100 000 pro Jahr auf. Typischer Unfallmechanismus für Unterarmschaftfrakturen ist ein direktes Anpralltrauma oder ein Unfall mit hoher Energieeinwirkung.

Vollständige Unterarmschaftfrakturen sollten aufgrund schlechter funktioneller Ergebnisse der konservativen Therapie generell operativ versorgt werden. Ausgenommen hiervon sind nicht dislozierte isolierte Frakturen der Ulna (sog. „Parierfraktur“), die meist auch unter konservativer Therapie zufriedenstellende Ergebnisse erzielen. Aktueller Therapiestandard ist die Kompressionsplattenosteosynthese bei einfachen Frakturen oder eine winkelstabile Plattenosteosynthese bei komplexeren Frakturen.

Um die hohen Ansprüche in Bezug auf Stabilität und Mobilität des Unterarms zu erhalten, ist ein komplexes Zusammenspiel der beteiligten anatomischen Strukturen erforderlich. An diesem Zusammenspiel sind neben Radius und Ulna auch das proximale und distale Radioulnargelenk sowie ligamentäre Strukturen wie die Membrana interossea beteiligt. Ziel der Behandlung von Unterarmfrakturen ist daher stets die Wiederherstellung der ursprünglichen anatomischen Verhältnisse. Bei komplexen Frakturen oder im Falle einer Revision kann sich der Operateur an anatomischen Landmarken wie bspw. dem radialen Bogen orientieren, der präoperativ auch am konventionellen Röntgenbild der betroffenen oder der Gegenseite ausgemessen werden kann ( $\triangleright$ Abb. 1). Hierbei muss allerdings streng der Vorteil von Aufnahmen der Gegenseite mit strahlenhygienischen Bedenken abgewogen werden.

Spezifische Komplikationen einer Unterarmschaftfraktur sind die verzögerte Frakturheilung, Ausbildung einer Pseudarthrose, die Verheilung der Fraktur in Fehlstellung (Malunion), heterotope Ossifikationen mit Fusion von Ulna und Radius (Synostose) sowie das erhöhte Risiko für eine Refraktur nach frühzeitiger Metallentfernung.

Pseudarthrosen von Unterarmfrakturen stellen eine besondere Herausforderung in der Behandlung dar, da die hohen Ansprüche an eine anatomische Rekonstruktion weiter erschwert werden. Abweichungen von der anatomischen Achse im Falle einer Unterarmfraktur können generell zu erheblichen Bewegungseinschränkungen des Unterarms und der Hand führen, die wiederum zu deutlichen Funktionsdefiziten führen können.

Ziel dieses Artikels ist es, die Ätiologie und Häufigkeit schlecht heilender Frakturen am Unterarm des Erwachsenen darzustellen und aktuelle Therapiestrategien in dieser Situation zusammenzufassen.

\section{Häufigkeit und Risikofaktoren verzöger- ter Frakturheilung am Unterarm}

Durch moderne Osteosyntheseverfahren können Heilungsraten bei Unterarmschaftfrakturen von ca. $98 \%$ er-

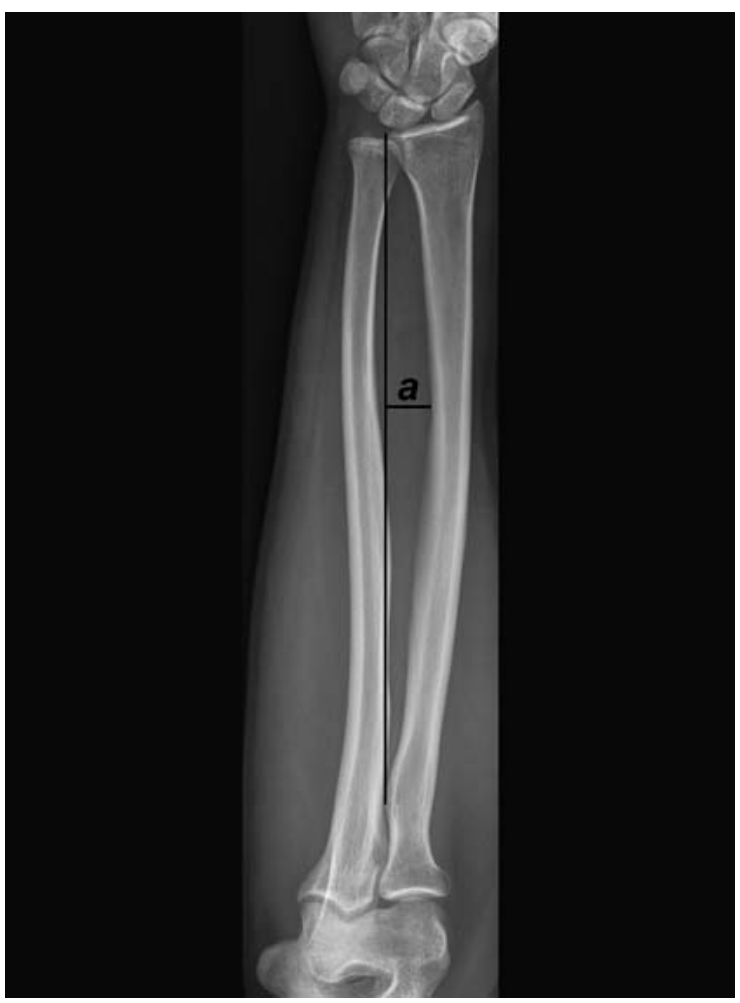

- Abb. 1 Der radiale Bogen (a) kann als anatomischer Parameter zur Wiederherstellung der anatomischen Verhältnisse dienen. Hierfür kann man sich unter Abwägung strahlenhygienischer Bedenken auch an der Gegenseite orientieren, indem man die maximale Höhe des Bogens im Seitenvergleich bestimmt und auf die Gegenseite spiegelt. 
reicht werden [1 -4]. Eine verzögerte Frakturheilung am Unterarm wurde in den größten Kollektiven von Unterarmfrakturen mit einer Häufigkeit von 0-5\% angegeben, Pseudarthrosen in 2-5,3\% [2,4-7]. Die meisten Pseudarthrosen am Unterarm sind oligotrophe oder atrophe Pseudarthrosen, nur ca. 9\% der Pseudarthrosen sind hypertroph [8].

Das Risiko für eine Pseudarthrose hängt von verschiedenen Faktoren wie der anatomischen Lokalisation der Fraktur, dem Verletzungsmuster, begleitenden allgemeinen Risikofaktoren und der verwendeten operativen Technik ab.

Historisch bedingt wird bei Unterarmfrakturen in der Literatur bereits bei der primären Verletzung eine Spongiosaplastik diskutiert. Grund hierfür war die Empfehlung von Anderson et al., die eine Spongiosaplastik auch bei kleineren Trümmerzonen empfohlen haben, wobei bereits in diesem Kollektiv kein evidenter Unterschied hinsichtlich der Konsolidierungsrate durch eine primäre Spongiosaplastik nachgewiesen werden konnte [5]. Auch in neueren Publikationen konnte kein eindeutiger Vorteil einer primären Spongiosaplastik zur Verminderung der Pseudarthroseraten gezeigt werden [1-4,9]. Aufgrund der fehlenden Evidenz kann daher eine primäre Spongiosaplastik nicht als Standard empfohlen werden.

\section{Verletzungsmuster}

Wie bei anderen Körperregionen ist auch am Unterarm das Verletzungsmuster entscheidend für die Ausbildung einer Pseudarthrose nach einer Fraktur. Die Häufigkeit einer Pseudarthrose hängt u.a. von der Lokalisation der Fraktur ab. Für isolierte Frakturen des Ulnaschaftes konnten Brakenbury et al. in ihrer Serie zeigen, dass über 70\% der 21 untersuchten Pseudarthrosen am mittleren Schaftdrittel vorkommen [10].

Ist eine Trümmerzone oder eine Dislokation über eine halbe Schaftbreite vorhanden, liegt die Pseudarthroserate deutlich höher $[9,10]$. Darüber hinaus konnte mehrfach gezeigt werden, dass die Pseudarthroserate bei offenen Frakturen gegenüber geschlossenen Frakturen deutlich erhöht ist [4,5]. Verletzungen mit höherenergetischem Trauma haben ebenfalls höhere Pseudarthroseraten [10].

\section{Risikofaktoren}

In der Literatur werden verschiedene Faktoren diskutiert, die das Risiko für eine Pseudarthrose allgemein erhöhen sollen. Hierzu zählen u.a. ein höheres Alter, Diabetes mellitus und starkes Übergewicht [11-13]. Ein höheres Risiko für eine schlechtere Knochenheilung konnte ebenfalls bei Rauchern an verschiedenen Lokalisationen nachgewiesen werden $[14,15]$. Spezifische Daten zu schlechterer Frakturheilung nach Unterarmfrakturen bei Rauchern gibt es zwar nicht, Chen et al. konnten aber bei 39 unter- suchten Patienten nach Verkürzungsosteotomie der Ulna höhere Raten einer verzögerten oder ausbleibenden Knochenheilung (30 vs. $0 \%$ ) bei Rauchern zeigen [16].

\section{Operative Faktoren}

Verschiedene Faktoren in der operativen Versorgung von Unterarmfrakturen können zu höheren Raten einer Pseudarthrose führen. Anderson et al. führten in ihrer großen Fallserie die aufgetretenen Pseudarthrosen ohne Nachweis eines Infekts auf technische Fehler in der Versorgung zurück [5]. Dabei war entweder die Plattenlänge falsch gewählt, die Platte nicht korrekt über der Fraktur zentriert oder die eingebrachten Schrauben waren zu nah an der Fraktur eingebracht [5]. Stern et al. beschrieben in ihrer Serie mit 64 Patienten weniger Pseudarthrosen bei Versorgung mit 5 oder mehr Schrauben [17]. Generell gilt bei der Frakturversorgung die offene Versorgung als ein Risikofaktor für die Entwicklung einer Pseudarthrose [13]. Ziel der primären operativen Versorgung von Frakturen am Unterarm ist aufgrund der engen anatomischen Korrekturgrenzen die primäre Knochenbruchheilung unter Umgehung einer ausgeprägten Kallusbildung. Diese kann, wenn möglich, durch eine stabile Versorgung unter Kompression der Frakturzone erreicht werden. Bleibt eine stabile Versorgung aus, besteht eine höhere Gefahr für eine verzögerte Frakturheilung. Allgemein sollte außerdem ein vorsichtiges perioperatives Weichteilmanagement bei der Frakturversorgung erfolgen und möglichst das Periost nicht mehr als notwendig abgelöst werden, um die Durchblutung der Frakturzone nicht zu kompromittieren. Der Stellenwert weichteilschonenderer Marknagelosteosynthesen ist noch nicht geklärt.

\section{Behandlungsstrategien bei verzögerter Frakturheilung am Unterarm}

\section{Allgemeine Strategien}

Prinzipiell bietet das „Diamond Concept“ eine Grundlage für die erforderlichen Faktoren im Falle einer problematischen Frakturheilung $[18,19]$. Zu beachten ist demnach, dass nicht nur die mechanische Stabilität, sondern auch biologische Faktoren wie vorhandene knochenbildende Zellen, Wachstumsfaktoren und eine erhaltene Durchblutung entscheidend für eine erfolgreiche Frakturheilung sind. Als Konsequenz sollte die Analyse der Ursache für die Pseudarthrose am Beginn der Therapie stehen und ein entsprechendes Therapiekonzept geplant werden.

Allgemein sind die wenigsten Pseudarthrosen am Unterarm hypertroph, der überwiegende Teil stellt sich als oligotrophe oder atrophe Pseudarthrose dar ( $\mathbf{A b b}$. 2) [8]. Meistens ist daher weniger eine fehlende mechanische Stabilität, sondern eine unzureichende biologische Aktivität für die Pseudarthrose verantwortlich. Die Sonderform 

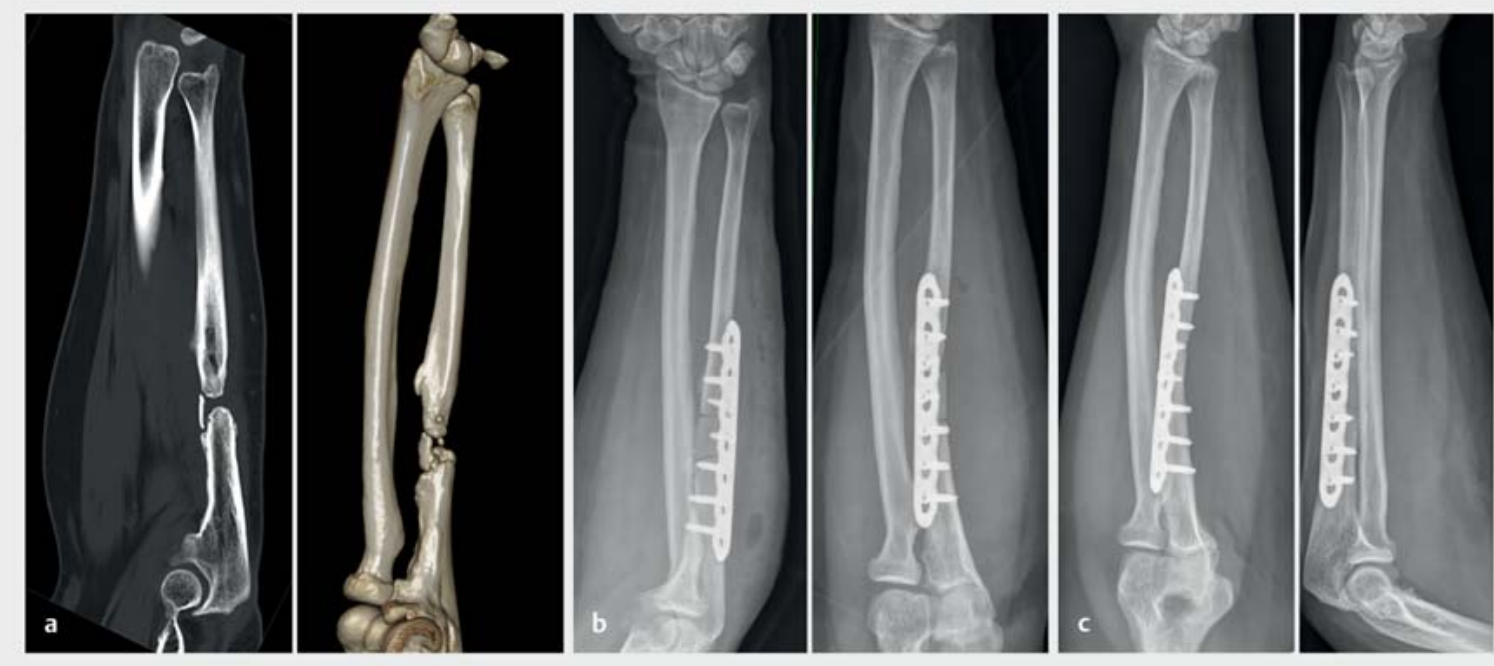

- Abb. 2 Atrophe Pseudarthrose nach Parierfraktur der Ulna. a CT-radiologische Bilder einer atrophen Pseudarthrose am proximalen Ulnaschaft. b Nach Augmentation mit einem Beckenkammspan. c Nach Ausheilung der Pseudarthrose.

stellt eine Infektpseudarthrose dar, die unten gesondert besprochen wird.

Für die konservative Therapie einer verzögerten Frakturheilung am Unterarm steht bspw. die extrakorporale Stoßwellentherapie (ESWT) zur Verfügung. Insgesamt sind die Ergebnisse dieses Verfahrens bei einer verzögerten Frakturheilung zwar ermutigend, die Qualität der verfügbaren Daten ist allerdings für eine abschließende Beurteilung unzureichend [20]. Bei nur minimalen Nebenwirkungen (Hämatome, petechiale Einblutungen) kann die Indikation allerdings großzügig gestellt werden.

Für die biologische Augmentation stehen primär generell verschiedene Arten der Augmentation zur Verfügung:

1. eine (autologe oder allogene) Spongiosaplastik,

2. die Interposition eines nicht vaskularisierten Spans und

3. die Interposition eines vaskularisierten Spans.

Daneben existieren besondere Verfahren, wie der Segmenttransport oder die Masquelet-Technik, die als Reserveverfahren in der Behandlung von Knochendefekten zum Einsatz kommen.

In der Literatur finden sich prinzipiell ermutigende Ergebnisse zur Therapie einer Pseudarthrose am Unterarm. Demnach können Pseudarthrosen am Unterarm erfolgreich mit einem Débridement der Pseudarthrosezone, einer stabilen Reosteosynthese, der biologischen Augmentation mit (autologem) Knochenmaterial und möglichst früher Mobilisation behandelt werden [21]. Kloen et al. konnten beispielsweise bei 47 Patienten mit 51 Pseudarthrosen des Unterarms nach einem Median von
7 Monaten alle Pseudarthrosen mithilfe dieser allgemeinen Prinzipien zur Ausheilung bringen [21]. Unklarheit besteht aber weiterhin in Bezug auf eine Strategie zur Art der knöchernen Augmentation.

\section{Strukturelle Augmentation vs. nicht strukturelle Augmentation}

Erste Berichte stammen von Nicoll et al., die bereits 1956 ihre Augmentationsstrategie mit einem Beckenkammspan beschrieben. Sie konnten mit dieser Strategie in allen Fällen eine knöcherne Konsolidierung erreichen. Zahlreiche weitere Arbeiten haben seither über die erfolgreiche Interposition eines kortikospongiösen Spans berichtet [22-28]. Späne werden von der Fibula, dem Beckenkamm oder als kortikaler Span von der Tibia genommen ( $>$ Abb. 3) [22-28]. Ring et al. konnten hingegen an einem Kollektiv von 35 Patienten zeigen, dass eine Ausheilung der Pseudarthrose auch nach alleiniger Interposition autologer Spongiosa erreicht werden kann [8].

Uneinheitlich wird auch über Ergebnisse bei der Verwendung autologer oder allogener Transplantate berichtet. Davis et al. berichten bspw. in einer Serie von 7 Infektpseudarthrosen über eine Interposition mit einem strukturellen Allograft [29]. Die Defektgröße lag dabei zwischen 2,3 und $10,4 \mathrm{~cm}$. Vier Patienten mussten allerdings nochmals mit autologem Graft augmentiert werden, um eine knöcherne Fusion an Radius oder Ulna zu erreichen [29]. Insgesamt liegt die berichtete primäre Ausheilungsrate nach (kortiko)spongiöser Augmentation zwischen 63 und $100 \%$ [22-28]. 

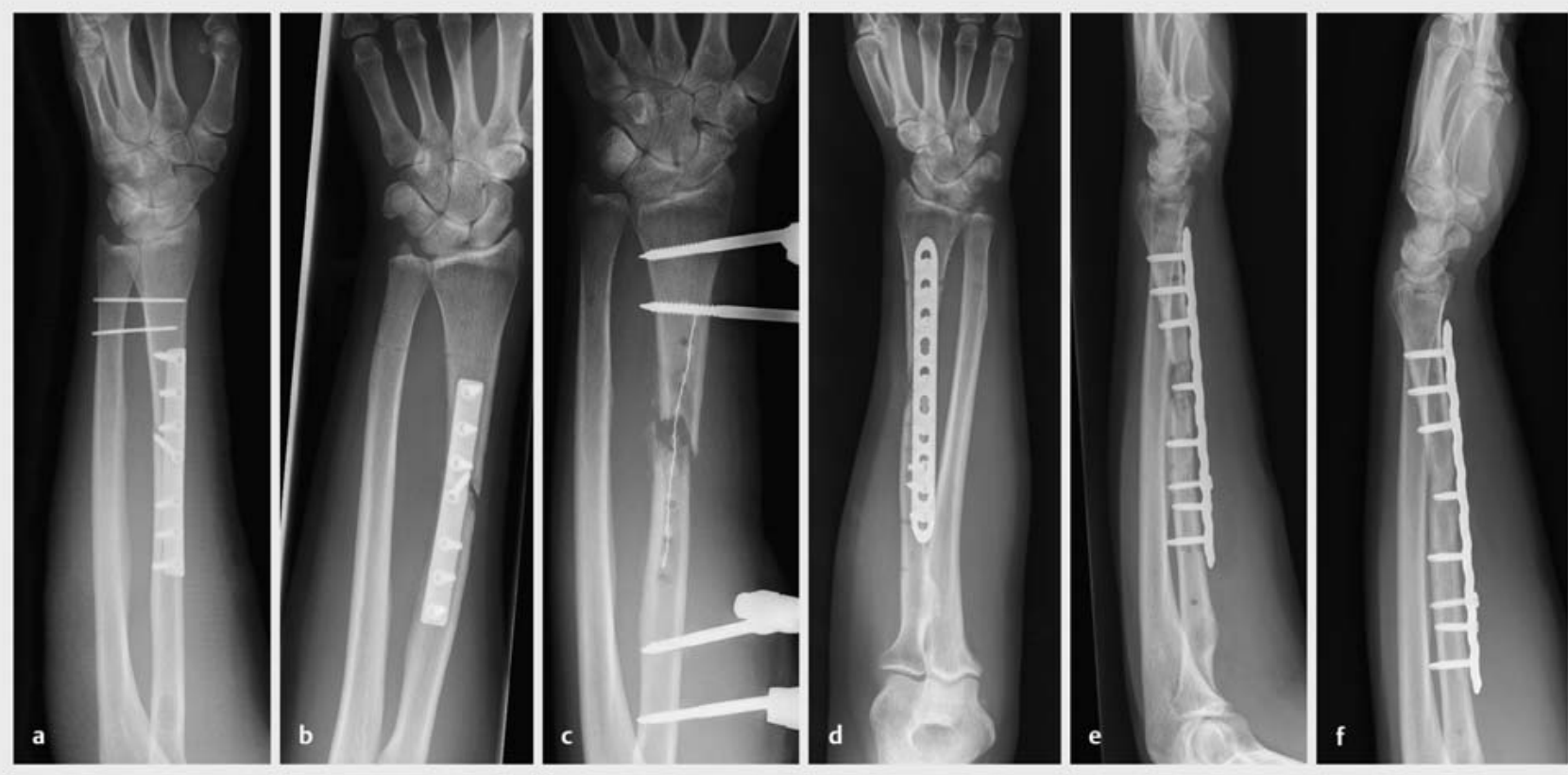

- Abb. 3 Infektpseudarthrose nach Galeazzi-Fraktur. a Primärversorgung mit Transfixation von distalem Radius und Ulna und Plattenosteosynthese des Radius. b Nach Metallentfernung der Transfixation. Eine zunehmende Konsolidierung ist auch 6 Wochen nach der Fraktur nicht sichtbar. c Metallentfernung, Débridement und Einlage einer Antibiotikakette bei Infektpseudarthrose am Radiusschaft. Die Fraktur kann temporär mit einem AO-Fixateur ruhig gestellt werden. $\mathbf{d}$ A.-p. Bild nach Spanaugmentation der Pseudarthrosezone. e Laterales Bild nach Spanaugmentation. f Erfolgreiche Behandlung mit Ausheilung der Pseudarthrose.

\section{Vaskularisierte Augmentation}

Für die Verwendung vaskularisierter Knocheninterponate existieren ebenfalls einige Fallserien [30-37]. Die häufigste Entnahmestelle für das Graft ist dabei die Fibula [30 - 32, 35 - 37]. Weitere Berichte beschreiben ein lokales Autograft von der distalen Ulna über die A. interosseus posterior oder vom distalen Radius $[33,34]$. Insgesamt wird auch bei der Verwendung vaskularisierter Grafts am Unterarm über gute Ergebnisse in Bezug auf die primäre knöcherne Ausheilung berichtet. Diese liegen zwischen 75 und $100 \%$ [30-37].

\section{Segmenttransport}

Über Techniken eines Segmenttransports am Unterarm existieren nur kleine Fallserien. Zhang et al. berichten bspw. über 16 Patienten mit einer Infektpseudarthrose, bei denen nach im Mittel 6,2 Monaten alle Pseudarthrosen zur Ausheilung gebracht werden konnten [38,39]. Für den Segmenttransport können unilaterale Fixateure $[38,40]$ oder Ringfixateure verwendet werden [39]. Die häufigste Indikation für einen Segmenttransport ist die Infektpseudarthrose oder eine Osteomyelitis [38-40].

\section{Masquelet-Technik}

Die Masquelet-Technik folgt dem Prinzip der „Membraninduktion“ [41]. Das bedeutet, dass nach Resektion eines knöchernen Segmentes zunächst temporär ein PMMASpacer (PMMA: Polymethylmethacrylat) eingebracht wird. Hierdurch wird ein Pseudoperiostschlauch um den Spacer generiert, der anschließend mit Spongiosa befüllt werden kann. Auch für die Verwendung dieser Technik bei einer Pseudarthrose am Unterarm existieren nur ein Fallbericht und eine kleine Fallserie, die allerdings beide gute Ergebnisse zeigen [42,43].

\section{Infektpseudarthrose}

Im Falle eines Infektes als Ursache für die ausbleibende Frakturheilung muss die Pseudarthrose debridiert, atrophes Gewebe abgetragen und infiziertes Material entfernt werden ( $\triangleright$ Abb.4). Eine unzureichend stabile Osteosynthese muss revidiert werden. Im Falle einer atrophen oder oligotrophen Pseudarthrose steht prinzipiell die biologische Augmentation im Vordergrund. Als Alternative zur Augmentation kann allerdings insbesondere in der Infektsituation ein Segmenttransport oder die Masquelet-Technik verwendet werden, da diese beiden Techniken keine Spaninterposition vorsehen, bei der sonst ein höheres Infektrisiko befürchtet wird.

\section{Differenzierte Indikation}

Prinzipiell spricht für eine alleinige (autologe) Spongiosaplastik im Vergleich zum autologen Knochenspan die geringere Entnahmemorbidität. Die Entnahme von Spänen an der Fibula oder am Beckenkamm geht häufig mit einer Deformierung und Schmerzen an der Entnahmestelle einher. 


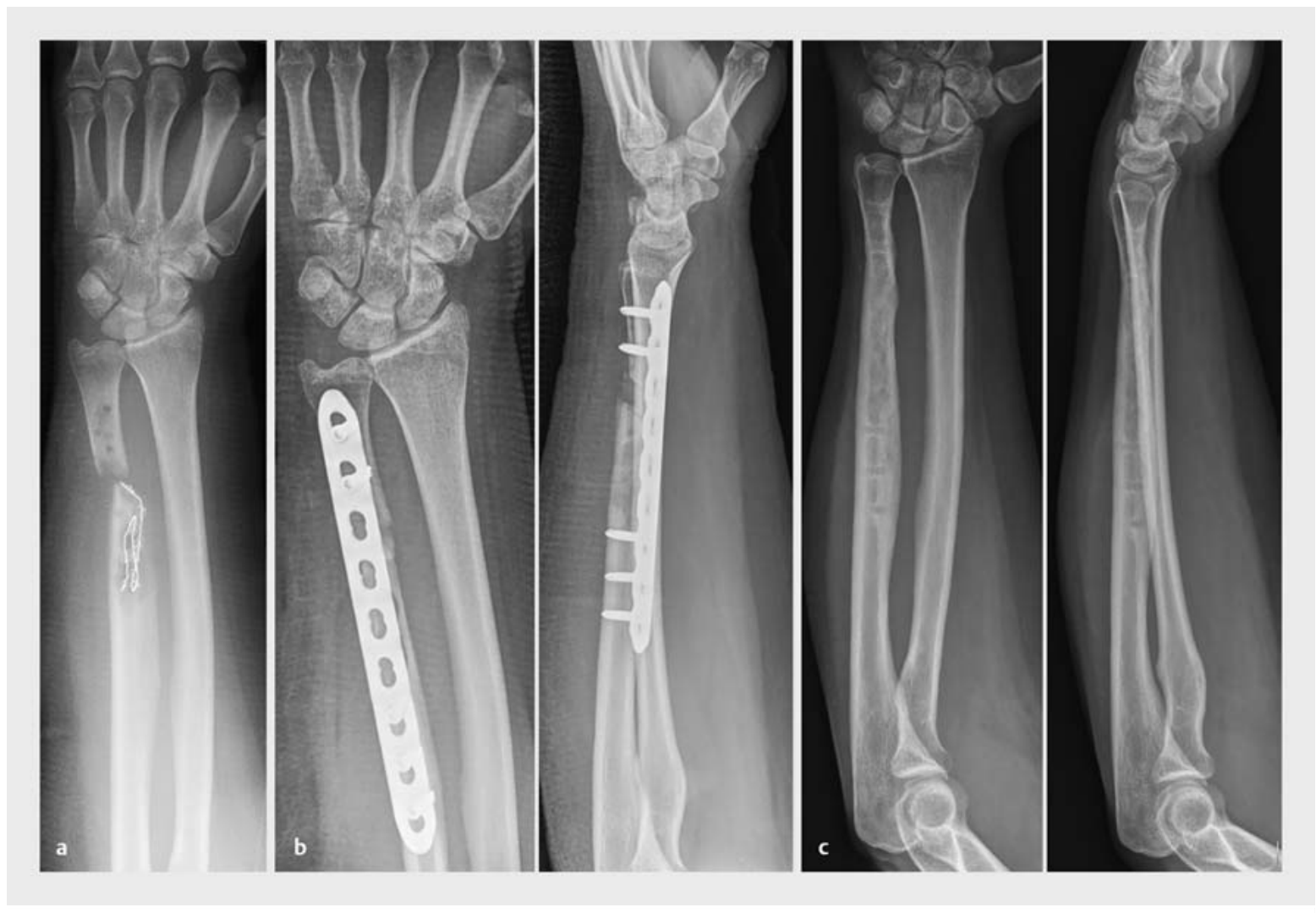

- Abb.4 Infektpseudarthrose nach Ulnaverkürzungsosteotomie. a Primär erfolgte die Metallentfernung und Einlage einer Antibiotikakette. b Nach Sanierung des Infekts Reosteosynthese mit 3,5-mm-LCP und Anlage autologer Spongiosa. c Ausheilungsbild nach Metallentfernung.

Allgemein wird empfohlen, dass kleinere Defekte mit einer alleinigen Spongiosaplastik aufgefüllt werden können, größere Defekte hingegen eher mit einem Knochenspan versorgt werden sollen [22, 24]. Dieser Empfehlung liegt die Annahme zugrunde, dass eine Spongiosaplastik ohne kortikalen Span nicht hinreichend Stabilität für die Versorgung größerer Defekte bietet. Eine Grenze für eine erfolgreiche Behandlung mit einer Spongiosaplastik bleibt allerdings unklar. Ring et al. konnten bspw. bei 35 Patienten mit Defekten zwischen 1 und $6 \mathrm{~cm}$ alle Pseudarthrosen mit einer Spongiosaplastik zur Ausheilung bringen, strukturelle Augmentate wurden nicht verwendet. Allgemein wird daher angenommen, dass bei stabiler Osteosynthese und guter Weichteildeckung eine strukturelle Augmentation bis $6 \mathrm{~cm}$ Defektlänge nicht erforderlich ist. Eine eindeutige Evidenz für diese Annahme existiert allerdings nicht.

Als Argument für vaskularisierte Späne gilt eine bessere Durchblutung des Spans, die zu einer besseren knöchernen Durchbauung und geringerer Resorption des Augmentats führen soll. Gegen vaskularisierte Späne sprechen allerdings deutlich längere Operationszeiten, die Notwendigkeit mikrochirurgischer Techniken und eine erhöhte Entnahmemorbidität. Als Grenze für die Verwendung nicht vaskularisierter Späne findet sich in der Literatur ebenfalls eine Defektlänge von $6 \mathrm{~cm}$. Davey et al. berichteten bspw. in ihrer Serie über eine fehlende Ausheilung der Pseudarthrose bei Verwendung eines nicht vaskularisierten Spans bei Versorgung eines Defekts $>10 \mathrm{~cm}$ Länge [24]. Allerdings gibt es keinerlei Evidenz, die für eine Verwendung einer Grenze von $6 \mathrm{~cm}$ spricht, sodass letztlich unklar bleibt, welche Defekte mit (kortiko)spongiöser Augmentation versorgt werden können und für welche Defekte aufwendigere, vaskularisierte Späne verwendet werden sollten [44].

Prinzipiell ist die biologische Aktivität von autologem gegenüber allogenem Material höher und damit eine erfolgreiche Behandlung wahrscheinlicher [45]. Allerdings fehlen auch für diese Annahme klare Behandlungsgrenzen oder Empfehlungen. In der eigenen Praxis wird (kortiko)spongiöses Material, wenn möglich, zunächst am Beckenkamm oder aus dem Femur entnommen. Die Entnahme aus dem Femur eignet sich besonders dann, wenn eine Entnahme aus dem Beckenkamm nicht genügt. Hierfür stehen spezielle Operationssysteme (Reamer-Irrigator-Aspirator [RIA], Fa. Synthes) zur Verfügung. Sollte 
eine autologe Spongiosaplastik nicht (mehr) möglich sein bzw. nicht ausreichen, wird allogenes Material (additiv) verwendet.

Die Behandlungen mit alternativen Verfahren wie dem Segmenttransport oder dem Masquelet-Verfahren sind insgesamt deutlich schlechter untersucht, sodass diese in der eigenen Praxis nur Reserveverfahren darstellen, sollte eine Augmentation nicht möglich sein oder eine Infektpseudarthrose vorliegen. Auch wenn diese Verfahren insgesamt aufgrund der langen Behandlungsdauer, der Verwendung unbequemer Fixateure oder geplanter Reoperationen unbeliebt erscheinen, bieten sie bei guten Heilungsraten den großen Vorteil der fehlenden (im Falle des Segmenttransportes) oder zumindest geringeren Entnahmemorbidität gegenüber strukturellen Augmentaten.

Bezogen auf das Osteosyntheseverfahren können im Rahmen einer Revision Plattenosteosynthesen oder Marknagelosteosynthesen verwendet werden. Im Falle einer fehlgeschlagenen Plattenosteosynthese kann auch ein Verfahrenswechsel auf eine Marknagelosteosynthese erwogen werden [46]. Dieser Überlegung liegen die positiven Erfahrungen aus der Marknagelosteosynthese von Tibiaschaftfrakturen zugrunde. Direkt vergleichende Daten zwischen intramedullären Implantaten und Plattenosteosynthesen sind nicht vorhanden, für die intramedullären Implantate wird aber eine geringere Rotationsstabilität und Kompression angenommen, bei allerdings deutlich geringerem Weichteiltrauma während der Implantation. Die unterlegene mechanische Stabilität und insgesamt nur sehr geringe Datenlage zum Einsatz der Marknagelosteosynthesen führt allerdings zu einem insgesamt zurückhaltenden Einsatz dieser Technik.

Eine ergänzende lokale medikamentöse Therapie kann aktuell nicht als Standard für die Behandlung von Pseudarthrosen am Unterarm empfohlen werden. Kommerziell erhältliche Wachstumsfaktoren wie BMP-2 und BMP-7 haben nur eine schwache Evidenz in der Behandlung von Pseudarthrosen langer Röhrenknochen und Wirbelkörperfusionen [47]. Zudem sind beide Substanzen nicht für die Anwendung am Unterarm zugelassen und nur eingeschränkt verfügbar. Konzentriertes Knochenmarkaspirat (BMAC) oder thrombozytenreiches Plasma (PRP) konnten in der lokalen Behandlung keinen signifikanten Vorteil zeigen $[48,49]$. Systemisch verabreichte Medikamente (wie bspw. Parathormon oder Strontiumranelat) könnten bei Patienten mit einer begleitenden Osteoporose hilfreich sein [50,51]. Weitere Ansätze wie die medikamentöse Blockierung spezifischer Signalwege, die für den Knochenabbau relevant sind, oder die Transplantation autologer $\mathrm{CD}_{3} 4^{+}$-Granulozyten auf eine lokal applizierte Matrix haben das experimentelle Stadium noch nicht verlassen, könnten aber möglicherweise zukünftig interessant werden [52-54]. Den häufig eingesetzten, nicht steroidalen antiinflammatorischen Medikamenten
(NSAIDs) wird ein negativer Effekt auf die Frakturheilung nachgesagt, eine abschließende Bewertung ist allerdings weiterhin aufgrund der widersprüchlichen Datenlage nicht möglich [55].

\section{Zusammenfassung und Ausblick}

Pseudarthrosen sind aufgrund der hohen funktionellen Erfordernisse am Unterarm besonders problematisch und bedürfen einer möglichst anatomischen Rekonstruktion. Dennoch sind bei richtiger Indikationsstellung gute Ergebnisse möglich. Nachdem der Grund für die Pseudarthrose untersucht wurde, kann durch ein Débridement der Pseudarthrose und stabile Versorgung die Pseudarthrose meist erfolgreich behandelt werden. Ein Infekt als Ursache für die Pseudarthrose sollte unbedingt ausgeschlossen werden. Sollte durch das Débridement der Pseudarthrose ein Knochendefekt entstehen, kann dieser, abhängig von der Größe, meist nicht strukturell (mit autologer oder allogener Spongiosa) oder strukturell (mit vaskularisiertem oder nicht vaskularisiertem Knochenspan) augmentiert werden. Reserveverfahren, wie der Segmenttransport oder die Masquelet-Technik, sind selten in der Primärbehandlung einer Pseudarthrose am Unterarm erforderlich.

Besonders bedeutsam für die Ausheilung einer Pseudarthrose ist neben der stabilen Versorgung allerdings ein vorsichtiges Weichteilmanagement. Eine ausgiebige subperiostale Präparation sollte vermieden werden und die Pseudarthrose ausreichend mit Weichteilen bedeckt sein.

In Zukunft könnten systematische oder lokale medikamentöse Therapieoptionen wie bspw. eine künstlich hergestellte Matrix hinzukommen, die die Ausheilungsraten weiter verbessern könnten und eine autologe Knochentransplantationen möglicherweise seltener erforderlich machen.

\section{Interessenkonflikt}

Die Autoren geben an, dass kein Interessenkonflikt besteht.

\section{Autorinnen/Autoren}

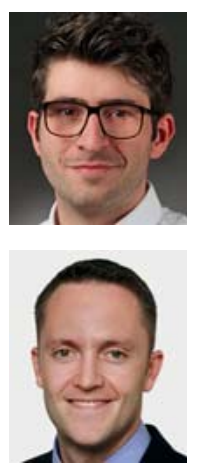

\section{Valentin Rausch}

Dr. med., Assistenzarzt, Universität Köln, Medizinische Fakultät und Universitätsklinik, Klinik für Orthopädie und Unfallchirurgie, Deutschland

\section{Michael Hackl}

Dr. med., Oberarzt, Universität Köln, Medizinische Fakultät und Universitätsklinik, Klinik für Orthopädie und Unfallchirurgie, Deutschland 


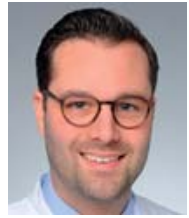

\section{Christian Ries}

PD Dr. med., Oberarzt, Universität Köln, Medizinische Fakultät und Universitätsklinik, Klinik für Orthopädie und Unfallchirurgie, Deutschland

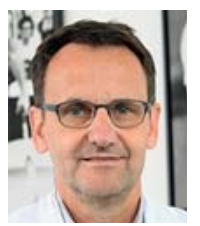

\section{Lars P. Müller}

Univ.-Prof. Dr. med., Leiter Schwerpunkt Unfall-, Hand- und Ellenbogenchirurgie, Universität Köln, Medizinische Fakultät und Universitätsklinik, Klinik für Orthopädie und Unfallchirurgie, Deutschland

\section{Korrespondenzadresse}

Valentin Rausch
Medizinische Fakultät und Universitätsklinik,
Klinik für Orthopädie und Unfallchirurgie
Universität Köln
Kerpener Straße 62
50937 Köln
Tel.: $0221 / 478-4802$
Fax: 0221/478-4835
rauschv@gmail.com

Literatur

[1] Schemitsch EH, Richards RR. The effect of malunion on functional outcome after plate fixation of fractures of both bones of the forearm in adults. J Bone Joint Surg Am 1992; 74: 10681078. doi:10.2106/00004623-199274070-00014

[2] Wei SY, Born CT, Abene A et al. Diaphyseal forearm fractures treated with and without bone graft. J Trauma 1999; 46: 1045-1048. doi:10.1097/00005373-199906000-00011

[3] Wright RR, Schmeling GJ, Schwab JP. The necessity of acute bone grafting in diaphyseal forearm fractures: a retrospective review. J Orthop Trauma 1997; 11: 288-294. doi:10.1097/ 00005131-199705000-00012

[4] Chapman MW, Gordon JE, Zissimos AG. Compression-plate fixation of acute fractures of the diaphyses of the radius and ulna. J Bone Joint Surg Am 1989; 71: 159-169

[5] Anderson LD, Sisk TD, Tooms RE et al. Compression-plate fixation in acute diaphyseal fractures of the radius and ulna. J Bone Joint Surg Am 1975; 57: 287-297

[6] Ross ERS, Gourevitch D, Hastings GW et al. Retrospective analysis of plate fixation of diaphyseal fractures of the forearm bones. Injury 1989; 20: 211-214. doi:10.1016/0020-1383 (89)90114-9

[7] Hadden WA, Reschauer R, Seggl W. Results of AO plate fixation of forearm shaft fractures in adults. Injury 1983; 15: 4452. doi:10.1016/0020-1383(83)90162-6

[8] Ring D, Allende C, Jafarnia K et al. Ununited diaphyseal forearm fractures with segmental defects. J Bone Joint Surg Am 2016; 86: 2440-2445. doi:10.2106/00004623-20041100000013

[9] Ring D, Rhim R, Carpenter C et al. Comminuted diaphyseal fractures of the radius and ulna: Does bone grafting affect nonunion rate? J Trauma 2005; 59: 436-440. doi:10.1097/01. ta.0000174839.23348.43

[10] Brakenbury PH, Corea JR, Blakemore ME. Non-union of the isolated fracture of the ulnar shaft in adults. Injury 1981; 12: 371-375. doi:10.1016/0020-1383(81)90005-X
[11] Ricci WM, Streubel PN, Morshed S et al. Risk factors for failure of locked plate fixation of distal femur fractures: an analysis of 335 cases. J Orthop Trauma 2014; 28: 83-89. doi:10.1097/ BOT.0b013e31829e6dd0

[12] Hernandez RK, Do TP, Critchlow CW et al. Patient-related risk factors for fracture-healing complications in the United Kingdom General Practice Research Database. Acta Orthop 2012; 83: 653-660. doi:10.3109/17453674.2012.747054

[13] Zura R, Mehta S, Della Rocca G] et al. Biological risk factors for nonunion of bone fracture. JBJS Rev 2016; 4: 1-12. doi:10.2106/JBJS.RVW.0.00008

[14] Pearson RG, Clement RGE, Edwards KL et al. Do smokers have greater risk of delayed and non-union after fracture, osteotomy and arthrodesis? A systematic review with meta-analysis. BMJ Open 2016. doi:10.1136/bmjopen-2015-010303

[15] Patel R, Wilson R, Patel P et al. The effect of smoking on bone healing: a systematic review. Bone Joint Res 2013; 2: 102-111. doi:10.1302/2046-3758.26.2000142

[16] Chen F, Osterman AL, Mahony K. Smoking and bony union after ulna-shortening osteotomy. Am J Orthop 2001; 30: 486-489

[17] Stern PJ, Drury WJ. Complications of plate fixation of forearm fractures. Clin Orthop Relat Res 1983; (175): 25-29

[18] Giannoudis PV, Einhorn TA, Marsh D. Fracture healing: The diamond concept. Injury 2007. doi:10.1016/50020-1383(08) 70003-2

[19] Rausch V, Seybold D, Königshausen M et al. Grundlagen der Knochenbruchheilung. Orthopade 2017; 46: 640-647. doi:10.1007/s00132-017-3449-8

[20] Willems A, van der Jagt OP, Meuffels DE. Extracorporeal shock wave treatment for delayed union and nonunion fractures: a systematic review. J Orthop Trauma 2019; 33: 97-103. doi:10.1097/BOT.0000000000001361

[21] Kloen P, Wiggers JK, Buihze GA. Treatment of diaphyseal nonunions of the ulna and radius. Arch Orthop Trauma Surg 2010; 130: $1439-1445$

[22] Moroni A, Rollo G, Guzzardella M et al. Surgical treatment of isolated forearm non-union with segmental bone loss. Injury 1997; 28: 497-504. doi:10.1016/S0020-1383(97)00044-2

[23] Spira E. Bridging of bone defects in the forearm with iliac graft combined with intramedullary nailing. J Bone Joint Surg $\mathrm{Br}$ 1954; 36: 642-646

[24] Davey PA, Simonis RB. Modification of the Nicoll bone-grafting technique for nonunion of the radius and/or ulna. J Bone Joint Surg Br 2002; 84: 30-33. doi:10.1302/0301-620X.84B1.11799

[25] Faldini C, Pagkrati S, Nanni M et al. Aseptic forearm nonunions treated by plate and opposite fibular autograft strut. Clin Orthop Relat Res 2009; 467: 2125-2134. doi:10.1007/s11999009-0827-5

[26] Dabezies E], Stewart WE, Goodman FG et al. Management of segmental defects of the radius and ulna. J Trauma 1971; 11: 778-788

[27] Barbieri CH, Mazzer N, Aranda CA et al. Use of a bone block graft from the iliac crest with rigid fixation to correct diaphyseal defects of the radius and ulna. J Hand Surg Br 1997; 22: 395-401

[28] Grace TG, Eversmann WW. Forearm fractures treatment by rigid fixation with early motion. J Bone Joint Surg Am 1980; 62: $433-438$

[29] Davis JA, Choo A, O'Connor DP et al. Treatment of infected forearm nonunions with large complete segmental defects using bulk allograft and intramedullary fixation. J Hand Surg Am 2016. doi:10.1016/j.jhsa.2016.05.021 
[30] Wood MB. Upper extremity reconstruction by vascularized bone transfers: results and complications. J Hand Surg Am 1987; 12: 422-427. doi:10.1016/S0363-5023(87)80017-5

[31] Safoury Y. Free vascularized fibula for the treatment of traumatic bone defects and nonunion of the forearm bones. J Hand Surg Br 2005; 30: 67-72

[32] Noaman HH. Management of upper limb bone defects using free vascularized osteoseptocutaneous fibular bone graft. Ann Plast Surg 2013; 71: 503-509. doi:10.1097/SAP.0b013e3182a1aff0

[33] Kamrani RS, Farhoud A, Nabian MH et al. Vascularized posterior interosseous pedicled bone grafting for infected forearm nonunion. J Hand Surg Eur Vol 2016; 41: 441-447. doi:10.1177/1753193415601041

[34] Pagnotta A, Taglieri E, Molayem I et al. Posterior interosseous artery distal radius graft for ulnar nonunion treatment. J Hand Surg Am 2012; 37: 2605-2610. doi:10.1016/j. jhsa.2012.09.004

[35] Jupiter JB, Gerhard HJ, Guerrero J et al. Treatment of segmental defects of the radius with use of the vascularized osteoseptocutaneous fibular autogenous graft. J Bone Joint Surg Am 1997; 79: 542-550

[36] Dell PC, Sheppard JE. Vascularized bone grafts in the treatment of infected forearm nonunions. J Hand Surg Am 1984; 9: 653-658. doi:10.1016/S0363-5023(84)80006-4

[37] Vicenti G, Maruccia M, Carrozzo M et al. Free vascularized osteoseptocutaneous fibular flap for radius shaft nonunion: the final solution when the iliac crest autograft fails. A case report. Injury 2018; 49: S63-S70. doi:10.1016/j.injury.2018.11.030

[38] Zhang Q, Yin P, Hao M et al. Bone transport for the treatment of infected forearm nonunion. Injury 2014; 45: 1880-1884. doi:10.1016/j.injury.2014.07.029.

[39] El-Mowafi H, Elalfi B, Wasfi K. Functional outcome following treatment of segmental skeletal defects of the forearm bones by Ilizarov application. Acta Orthop Belg 2005; 71: 157-162

[40] Liu T, Liu Z, Ling L et al. Infected forearm nonunion treated by bone transport after debridement. BMC Musculoskelet Disord 2013; 14: 273. doi:10.1186/1471-2474-14-273

[41] Masquelet AC, Begue T. The concept of induced membrane for reconstruction of long bone defects. Orthop Clin North Am 2010; 41: 27-37. doi:10.1016/j.ocl.2009.07.011

[42] Walker M, Sharareh B, Mitchell SA. Masquelet reconstruction for posttraumatic segmental bone defects in the forearm. J Hand Surg Am 2019; 44: 342.e1-342.e8. doi:10.1016/j. jhsa.2018.07.003

[43] Micev AJ, Kalainov DM, Soneru AP. Masquelet technique for treatment of segmental bone loss in the upper extremity. J Hand Surg Am 201540:593-598. doi:10.1016/j.jhsa.2014. 12.007
[44] Allsopp B], Hunter-Smith DJ, Rozen WM. Vascularized versus nonvascularized bone grafts: what is the evidence? Clin Orthop Relat Res 2016; 474: 1319-1327. doi:10.1007/s11999016-4769-4

[45] Finkemeier CG. Bone-grafting and bone-graft substitutes. J Bone Joint Surg Am 2002; 84: 454-464. doi:10.2106/ 00004623-200203000-00020

[46] Hofmann A, Hessmann MH, Rudig L et al. Intramedulläre Nagelosteosynthese der Ulna in der Revisionschirurgie. Unfallchirurg 2004; 107: 583-592. doi:10.1007/s00113-004-0790-2

[47] Garrison K, Shemilt I, Donell S et al. Bone morphogenetic protein (BMP) for fracture healing in adults. Cochrane Database Syst Rev 2010; (6): CD006950. doi:10.1002/14651858. CD006950.pub2

[48] Griffin XL, Wallace D, Parsons N et al. Platelet rich therapies for long bone healing in adults. Cochrane Database Syst Rev 2012; (7): CD009496. doi:10.1002/14651858.CD009496. pub2

[49] Griffin XL, Smith CM, Costa ML et al. The clinical use of platelet-rich plasma in the promotion of bone healing: a systematic review. Injury 2009; 40: 158-162. doi:10.1016/j.injury.2008. 06.025

[50] Alegre DN, Ribeiro C, Sousa C et al. Possible benefits of strontium ranelate in complicated long bone fractures. Rheumatol Int 2012; 32: 439-443. doi:10.1007/s00296-010-1687-8

[51] Pietrogrande L, Raimondo E. Teriparatide in the treatment of non-unions: Scientific and clinical evidences. Injury 2013; 44 (Suppl. 1): S54-S57. doi:10.1016/S0020-1383(13)70013-5

[52] Kuroda R, Matsumoto T, Niikura T et al. Local transplantation of granulocyte colony stimulating factor-mobilized CD34+ cells for patients with femoral and tibial nonunion: pilot clinical trial. Stem Cells Transl Med 2014; 3: 1079-1089. doi:10.5966/sctm.2014-0007

[53] Ke HZ, Richards WG, Li X et al. Sclerostin and Dickkopf-1 as therapeutic targets in bone diseases. Endocr Rev 2012; 33: 747-783. doi:10.1210/er.2011-1060

[54] Ominsky MS, Boyce RW, Li X et al. Effects of sclerostin antibodies in animal models of osteoporosis. Bone 2016; 96: 6375. doi:10.1016/j.bone.2016.10.019

[55] Marquez-Lara A, Hutchinson ID, Nuñez F et al. Nonsteroidal anti-inflammatory drugs and bone-healing. JBJS Rev 2016; 4: 1. doi:10.2106/JBJS.RVW.0.00055

\section{Bibliografie}

DOI https://doi.org/10.1055/a-0898-4402

OP-JOURNAL 2019; 35: 283-290 @ Georg Thieme Verlag KG Stuttgart · New York ISSN 0178-1715 